\title{
SHORT COMMUNICATION A Theory of Bubbles in Ion-implanted Structures
}

\author{
W. KINSNER \\ Department of Electrical Engineering and Industrial Applications of Microelectronics Centre, University of \\ Manitoba, Winnipeg, Manitoba, Canada R3T 2N2
}

Ion implantation was well established in the semiconductor technology. It could also be used for magnetic bubble technology. The original purpose of ion implantation in bubble materials had been to suppress the undesired hard bubbles. The formation of an implanted layer at the surface of negativemagnetostriction garnets appeared to be the best method of implementing the relatively old contiguous disc bubble propagating circuit of Bobeck and Della Torre. Proper selection of the parameters of the ion-implanted propagator, such as the depth, width, shape of the propagator walls, dopant concentration and profile, improves static and dynamic stability of the bubbles, their operating margins, packing density and power consumption.

This paper presented a mathematical model of bubbles inside straight side-wall ion-implanted channels. The model assumed a uniform dopant profile. The magnetizations in both the implanted and non-implanted material were assumed to be uniaxial and at saturation values. The ion-implantation changed the value of the saturation magnetization in the implanted material. This change led to a net magnetic charge at the interface between the implanted and non-implanted material. This magnetic charge distribution induced further magnetic charges on the side walls of the channel. The static magnetic pole distributions were affected by the presence of a bubble within the channel. The bubble induced other poles on the channel walls. All the poles affected, in turn, the geometry and the position of the bubble. The model included changes of the in-plane susceptibility and domain wall energy. The geometry, position and energy of the bubbles could then be computed. The computations were based on a variational formulation of an objective function. The process led to a minimum energy state of a generally deformable magnetic domain within the channel. Calculated results and conclusions concerning the stable energy, geometry and location of the bubbles were given and compared to other published theoretical and experimental results. 

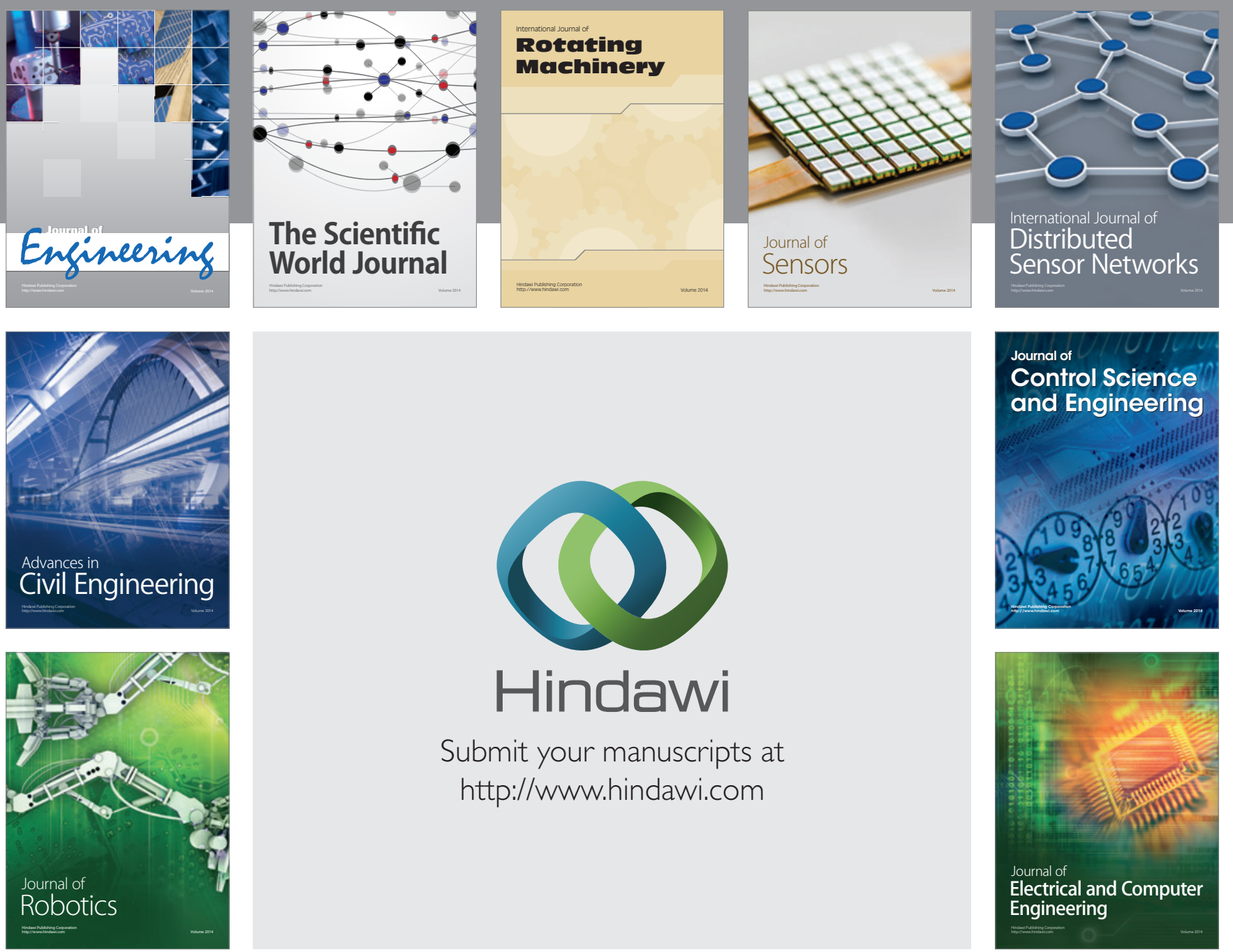

Submit your manuscripts at

http://www.hindawi.com
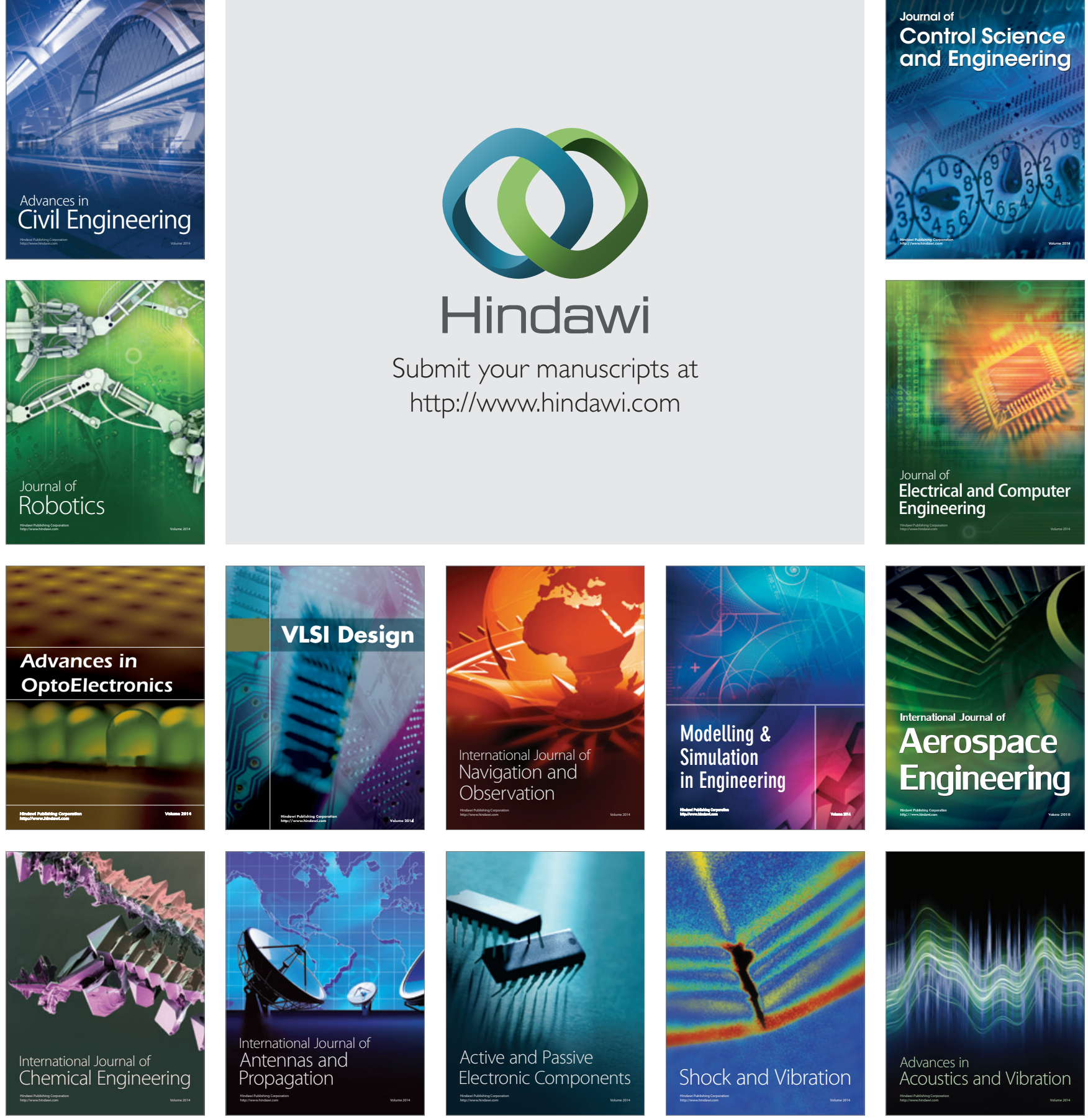